\title{
Polarized Light Propagation Through Tissue Phantoms
}

\author{
V. Sankaran, J.T. Walsh, D.J. Maitland
}

This article was submitted to

The International Society for Optical Engineering Photonics West, San Jose, CA, January 26, 2000

\section{February 8, 2000}

\section{U.S. Department of Energy}

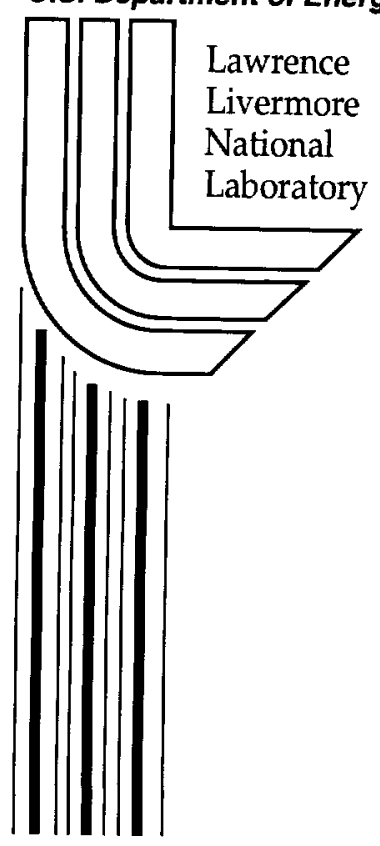




\section{DISCLAIMER}

This document was prepared as an account of work sponsored by an agency of the United States Government. Neither the United States Government nor the University of California nor any of their employees, makes any warranty, express or implied, or assumes any legal liability or responsibility for the accuracy, completeness, or usefulness of any information, apparatus, product, or process disclosed, or represents that its use would not infringe privately owned rights. Reference herein to any specific commercial product, process, or service by trade name, trademark, manufacturer, or otherwise, does not necessarily constitute or imply its endorsement, recommendation, or favoring by the United States Government or the University of California. The views and opinions of authors expressed herein do not necessarily state or reflect those of the United States Government or the University of California, and shall not be used for advertising or product endorsement purposes.

This is a preprint of a paper intended for publication in a journal or proceedings. Since changes may be made before publication, this preprint is made available with the understanding that it will not be cited or reproduced without the permission of the author.

This report has been reproduced directly from the best available copy.

Available electronically at http://www.doe.gov/bridge

Available for a processing fee to U.S. Department of Energy and its contractors in paper from

U.S. Department of Energy

Office of Scientific and Technical Information

P.O. Box 62

Oak Ridge, TN 37831-0062

Telephone: (865) 576-8401

Facsimile: (865) 576-5728

E-mail: reports@adonis.osti.gov

Available for the sale to the public from

U.S. Department of Commerce

National Technical Information Service

5285 Port Royal Road

Springfield, VA 22161

Telephone: (800) 553-6847

Facsimile: (703) 605-6900

E-mail: orders@ntis.fedworld.gov

Online ordering: http://www.ntis.gov/ordering.htm

\section{OR}

Lawrence Livermore National Laboratory

Technical Information Department's Digital Library

http://www.llnl.gov/tid/Library.html 


\title{
Polarized light propagation through tissue and tissue phantoms \\ Vanitha Sankaran* ${ }^{\text {a,b }}$, Joseph T. Walsh, Jr., Duncan J. Maitland ${ }^{\mathrm{b}}$ \\ ${ }^{a}$ Northwestern University, Evanston, IL 60208 \\ ${ }^{b}$ Lawrence Livermore National Laboratory, Livermore, CA 94551
}

\begin{abstract}
We show that standard tissue phantoms can be used to mimic the intensity and polarization properties of tissue. Polarized light propagation through biologic tissue is typically studied using tissue phantoms consisting of dilute aqueous suspensions of microspheres. The dilute phantoms can empirically match tissue polarization and intensity properties. One discrepancy between the dilute phantoms and tissue exist: common tissue phantoms, such as dilute Intralipid and dilute 1$\mu \mathrm{m}$-diameter polystyrene microsphere suspensions, depolarize linearly polarized light more quickly than circularly polarized light. In dense tissue, however, where scatterers are often located in close proximity to one another, circularly polarized light is depolarized similar to or more quickly than linearly polarized light. We also demonstrate that polarized light propagates differently in dilute versus densely packed microsphere suspensions, which may account for the differences seen between polarized light propagation in common dilute tissue phantoms versus dense biologic tissue.
\end{abstract}

\section{KEYWORDS}

Degree of polarization, Stokes polarimetry, multiple scattering, tissue phantom

\section{INTRODUCTION}

Optical imaging through highly scattering media is a problem that has been studied extensively in a number of fields, including atmospheric (1) and ocean optics (2), and more recently, biomedical optics (3-6). As photons travel through a turbid material, they can be unscattered or weakly scattered and provide information about the sample structure, or they can be highly scattered and contribute to the signal noise. Optical imaging can be enhanced through the discrimination and rejection of multiply scattered from weakly scattered photons. There are many such discrimination methods, including timeand frequency-domain techniques $(3,4)$, trans-illumination laser computed tomography (5), and confocal detection (6). Polarimetry, in which the polarization state of light incident on a sample is compared with the polarization state of light exiting the sample, has recently been under much study as another method of discrimination (7-10). Polarization-based discrimination is based on the premise that ballistic photons retain their initial polarization state whereas diffuse photons do not; thus, multiply scattered photons can be gated out based on their low degree of polarization.

Various studies have been conducted on how polarized light propagates in different tissue phantoms, often using aqueous suspensions of polystyrene microspheres (7-10). Based on the results from these investigations, it has been predicted that circularly polarized light may survive through more scattering events than linearly polarized light in an anisotropically scattering medium such as tissue $(7,8)$. However, we have previously shown that this is not necessarily the case (9). Typically, phantoms are chosen because their absorption and scattering properties match those of tissue; this does not necessarily imply that the polarization properties of the phantom match those of tissue. We have shown that simply matching the gross scattering coefficient of a phantom to a given tissue does not imply that polarized light will propagate similarly in the two samples (9). In this study, we show that both the intensity and polarization properties of tissue can be match using dilute polystyrene phantoms. The microsphere size, density and pathlength must be independently varied to empirically match the optical properties of tissue. Although this method is pragmatic, the resulting phantoms do not replicate the preferential propagation of linearly versus circularly polarized light in tissue. We show that dense microsphere phantoms correctly match the preferential propagation of linearly polarized light. We begin with a comparison of how linearly and circularly polarized light propagate in two different tissues, blood, which contains dilute spherical and quasispherical scatterers and myocardium tissue, which contains dense quasi-spherical scatterers. We then explain these results

\footnotetext{
"Correspondence: Email: sankaran1@IInl.gov; Telephone: (925)424-3101; FAX: (925)424-2778
} 
using tissue phantoms consisting of polystyrene microspheres suspended in water. The effects of scatterer size, phantom pathlength and density on polarimetric measurements are presented.

\section{THEORY}

\subsection{DEGREE OF POLARIZATION}

Degree of polarization is a term used to quantify the fraction of light that has retained its initial polarization after traveling through a turbid material. A degree of polarization of 1 corresponds to completely polarized light whereas a degree of polarization of 0 corresponds to completely unpolarized light. In this study, the propagation of linearly and circularly polarized light through a sample is described using Stokes vector measurements. The Stokes vector, $\mathbf{S}$, of light exiting a sample is a $4 \times 1$ vector that describes completely the polarization state of the collected light:

$$
\vec{S}=\left[\begin{array}{c}
I \\
Q \\
U \\
V
\end{array}\right],
$$

where $I$ is the total intensity, $Q$ is the difference between horizontal and vertical linearly polarized components, $U$ is the difference between $+45^{\circ}$ and $-45^{\circ}$ linearly polarized components, and $V$ is the difference between right and left circularly polarized components.

Values for the degree of polarization are calculated from each Stokes vector as:

$$
\begin{aligned}
& p_{L}(Q, U)=\text { Degree of linear polarization }=\frac{\sqrt{Q^{2}+U^{2}}}{I}, \\
& p_{C}(V)=\text { Degree of circular polarization }=\frac{\sqrt{V^{2}}}{I}, \text { and } \\
& p(Q, U, V)=\text { Total degree of polarization }=\frac{\sqrt{Q^{2}+U^{2}+V^{2}}}{I} .
\end{aligned}
$$

\subsection{RAYLEIGH AND MIE SCATTERERS}

In order to compare studies conducted on different samples with different incident wavelengths, it is often useful to speak in terms of Rayleigh and Mie scatterers. Mie scattering applies to particles that are large compared to the incident wavelength whereas the Rayleigh approximation to Mie theory applies to particles that are small compared to the incident wavelength. The relative size parameter, usually notated as $\alpha$ or $\mathrm{k}_{\mathrm{a}}$, can be used to relate the diameter of a scatterer, $\mathrm{d}$, to the incident wavelength, $\lambda(10)$ :

$$
\alpha=\frac{\pi \mathrm{d}}{\pi}
$$

(2)

Since the scattering of electromagnetic radiation scales linearly to the relative size parameter, $\alpha$ can be used to compare studies with different scatterer sizes and incident wavelengths. For small scatterers $(\alpha \leq \pi / 10)$, the amplitude of the scattered wavelets is small; thus the infinite series solutions giving the scattered amplitude and phase can be approximated by a sum 
of only the first few terms in the series. Scattering by these small particles is usually called Rayleigh scattering and is a subset of Mie theory, which is used to describe larger scatterers $(\alpha>\pi / 10)$.

\section{METHODS}

\subsection{EXPERIMENTAL SETUP}

A He-Ne laser (Melles Griot Inc., 05LHP925) emitting a collimated 1.5-mm 1/ $\mathrm{e}^{2}$ diameter beam of 632.8-nm-radiation was passed through a chopper operating at $2.78 \mathrm{kHz}$ (Stanford Research Systems, SR540), a linear polarizer (LP; Melles Griot Inc., 03FPG009), and into the sample. For circularly polarized light incident on the sample, a quarter-wave plate (QWP; Meadowlark Optics, NQM-100-633) was inserted between the polarizer and the sample. Light emerging from the sample was collected with a 55-mm-focal length, F/2.8 camera lens and passed through subsequent polarizing optics. An iris in the camera lens rejected light scattered beyond a full angle of $22.6^{\circ}$; this ensured that the collected light that was passed through the subsequent polarization optics fell within the acceptance angle of each element. The collected light was first passed through a variable-wave plate (VWP; Meadowlark Optics, LRC-100) oriented at $45^{\circ}$ to the horizontal, whose retardance, $\Delta$, was controlled by a voltage input. The light exiting the VWP was passed through a photoelastic modulator (PEM; Hinds Instruments, PEM-90) oriented at $0^{\circ}$ followed by a linear polarizer (LP; Melles Griot Inc., 03FPG009) oriented at $45^{\circ}$. The PEM is a wave plate whose retardance, $\delta=\delta_{0} \cos (\omega \mathrm{t})$, modulated between $\phi \delta_{0}$ at $\omega=2 \pi(50 \mathrm{kHz})$, thus providing the reference signal for lock-in detection. The collection optics, VWP, PEM and LP constituted the analysis section of the system. All signal components were measured with a $\$ 15 \mathrm{~V}$ photoconductive detector (Hinds Instruments, DET-90-002) and a lock-in amplifier (Stanford Research Systems, SR810). To simplify the signal analysis, $\delta_{0}$ was set to 138 ; thus, $\mathrm{J}_{0}\left(\delta_{\mathrm{o}}\right)=0$. To isolate $\mathrm{U}_{\text {sample }}$ and $\mathrm{V}_{\text {sample }}, \Delta$ was set to 0 中; $\mathrm{U}_{\text {sample }}$ was then measured at $2 \omega$ and $\mathrm{V}_{\text {sample }}$ was measured at $1 \omega$. Similarly, when $\Delta$ was set to 90 , $U_{\text {sample }}$ was measured at $2 \omega$ and $\mathrm{Q}_{\text {sample }}$ was measured at $1 \omega$. $I_{\text {sample }}$ was measured at the chopper frequency. The measurement technique used here is similar to that described by Schmitt et al. (7).

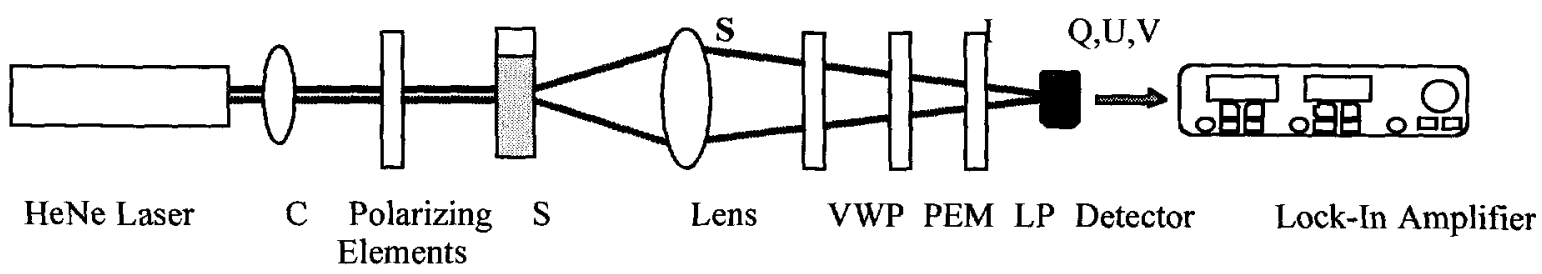

Figure 1. The experimental setup consists of a He-Ne laser, a chopper (C) operating at $2.78 \mathrm{kHz}$, polarizing elements for the light incident on the sample (S), a variable-wave plate (VWP), a photoelastic modulator (PEM) operating at $50 \mathrm{kHz}$, a linear polarizer (LP), a photodetector and a lock-in amplifier. The maximum degree of polarization with no sample present was 1.0001 .

\subsection{SAMPLES}

Depolarization by biologic scatterers was studied using porcine blood and porcine myocardial tissue. Each tissue was taken from freshly sacrificed 12 -month-old slaughterhouse pigs and stored at $5{ }^{\circ} \mathrm{C}$ prior to experimentation. The samples were warmed to room temperature $2 \mathrm{~h}$ before each experiment and were used within $48 \mathrm{~h}$ post mortem. Whole blood was heparinized within $1 \mathrm{~h}$ post mortem. Samples of whole blood were placed in a glass cuvette with a pathlength ranging from 0.1 to $5 \mathrm{~mm}$ for each experiment. Sections of myocardium with an average thickness ranging from 0.5 to $3.0 \mathrm{~mm}$ were mounted between glass slides for each experiment.

Depolarization in the tissue phantoms was studied using monodisperse suspensions of $0.102 \mu \mathrm{m}, 0.99 \mu \mathrm{m}$, and $0.99 \mu \mathrm{m}$ diameter polystyrene microspheres in water (Polysciences Inc., $\mathrm{n}_{\text {sphere }}=1.59, \mathrm{n}_{\text {medium }}=1.33$ ). Two different experiments were performed with these suspensions. First, for a given size of spheres, a fixed concentration of scatterers was chosen and prepared. Each sample was diluted with distilled water to a concentration ranging from 0.25 to $2.5 \%$, where independent, uncorrelated scattering could be assumed. Samples were placed in cuvettes with pathlengths ranging from 0.1 to $2 \mathrm{~cm}$; the 
degree of polarization for each sample was measured as a function of cuvette pathlength. Second, for a given size of spheres, a fixed cuvette pathlength was used to make degree of polarization measurements as a function of increasing scatterer concentration. The range of concentrations investigated spanned both dilute, independent scattering and dense, dependent scattering. Concentrations of spheres greater than $2.5 \%$ (the initial concentration obtained from the manufacturer) were obtained by centrifuging the sample so that the particles would settle and then decanting the excess water. Spheres with a diameter smaller than $0.48 \mu \mathrm{m}$ have a prohibitively long settling time and thus could not be studied here. The dense suspensions were sonicated to prevent clumping of the spheres and stirred before each measurement. Static repulsion between the anionically charged spheres should ensure a minimal distance between each sphere and its nearest neighbors; however this could not be verified experimentally. For concentrations of spheres less than $2.5 \%$, the suspension was diluted with distilled water. Samples were placed in a 1-cm-pathlength glass cuvette with flat parallel walls for experimentation.

\section{RESULTS \& DISCUSSION}

\subsection{BIOLOGIC TISSUE}

Blood was first used to investigate polarized light propagation through the simplest case of dilute, near-spherical scatterers. Degree of polarization results as a function of cuvette pathlength are shown in Figure 2. Blood is a fluid tissue that consists of various cells; one cubic milliliter of blood contains roughly 5,000,000 biconcave disk-like erythrocytes $(7 \mu \mathrm{m}$ in diameter), approximately 7,000 spherical leukocytes (8-18 $\mu \mathrm{m}$ in diameter), and between 150,000 and 300,000 biconvex disk-like platelets $(2-4 \mu \mathrm{m}$ in diameter), floating freely and separated from one another by plasma $(11,12)$. The scatterers in blood predominately fall into the Mie scattering regime for the incident $633-\mathrm{nm}$ radiation used here. For each sample of whole blood investigated, linearly polarized light is depolarized more than circularly polarized light.

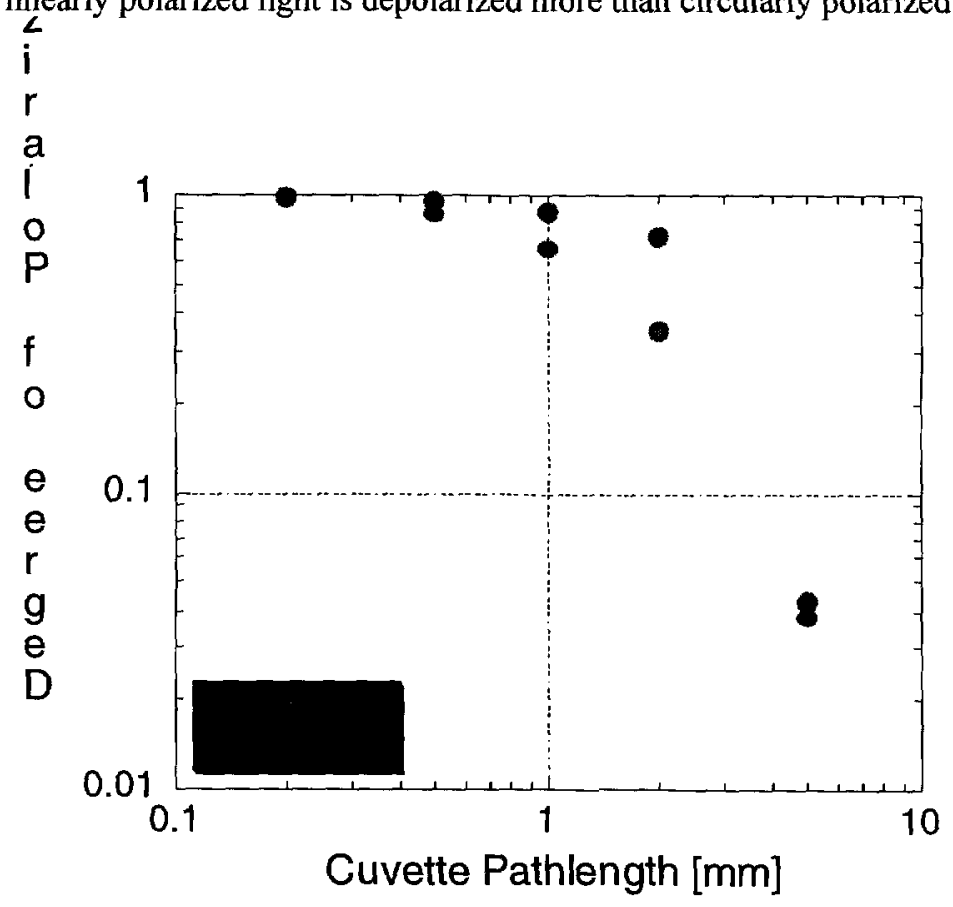

Figure 2. The degree of linear and circular polarization in whole porcine blood as a function of cuvette pathlength.

Myocardial tissue was used next to study polarized light scattering by densely packed near-spherical scatterers. Degree of polarization results as a function of tissue thickness are shown in Figure 3. Muscle cells range in size, with a mature muscle cell having the largest diameter of 1-3 $\mu \mathrm{m}$. Although the scatterers in myocardial tissue span the Rayleigh and Mie regimes, the structures are predominantly Mie-sized. For each tissue sample investigated, circularly polarized light is depolarized more than linearly polarized light, in contrast to the results seen in blood. 


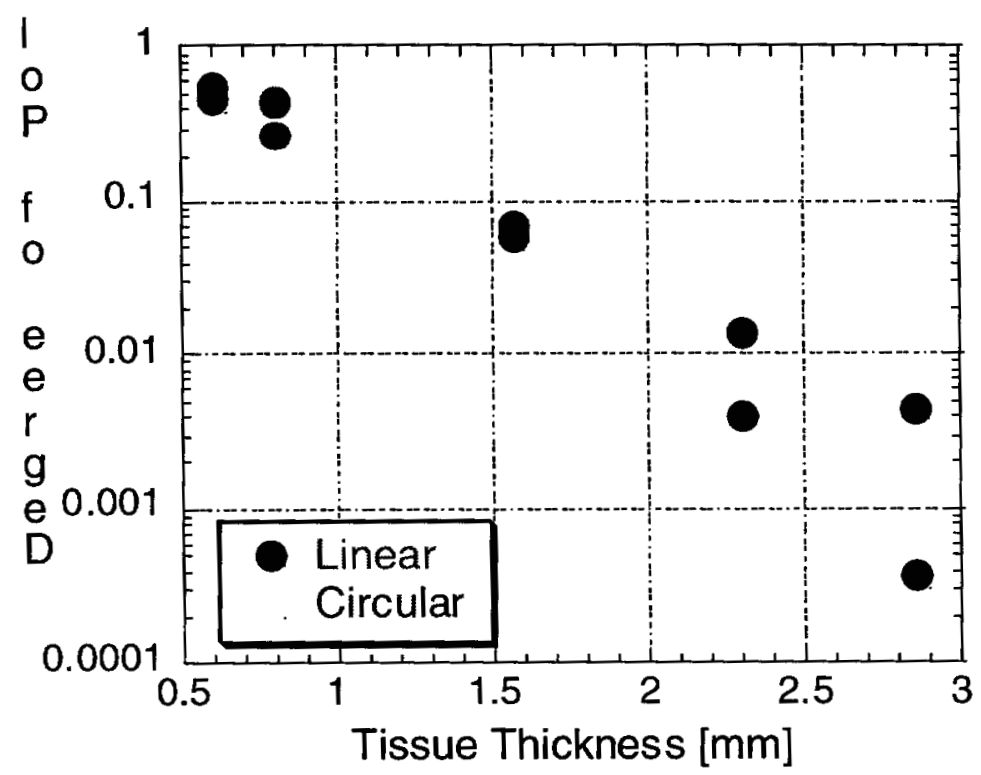

Figure 3. The degree of linear and circular polarization in sections of porcine myocardial tissue as a function of tissue thickness.

\subsection{TISSUE PHANTOMS: SIZE EFFECT}

Figure 4 compares the depolarization and intensity attenuation of three porcine tissues (whole blood, myocardium and adipose) and two dilute phantoms ( 0.1 and $0.99 \mu \mathrm{m}$ diameter microspheres). The intensity-attenuation/depolarization space shown in Figure 4 allows for a direct comparison between the phantoms and tissues relative to the important dependent variables. That is, the phantoms should mimic both the depolarization and intensity attenuation of tissue for it to be considered a reasonable phantom. For any given phantom microsphere size, we did not find that any phantom could well represent tissue if only one of two remaining independent variables were varied (pathlength \& density). However, if both pathlength and density are varied, we found that any phantom could be made to replicate a large area of the intensityattenuation/depolarization space. Figure 5 shows an example of this for $0.99 \mu \mathrm{m}$ microspheres. Via this empirical method, dilute microsphere phantoms could be made to replicate the polarization and intensity properties of any desired tissue.

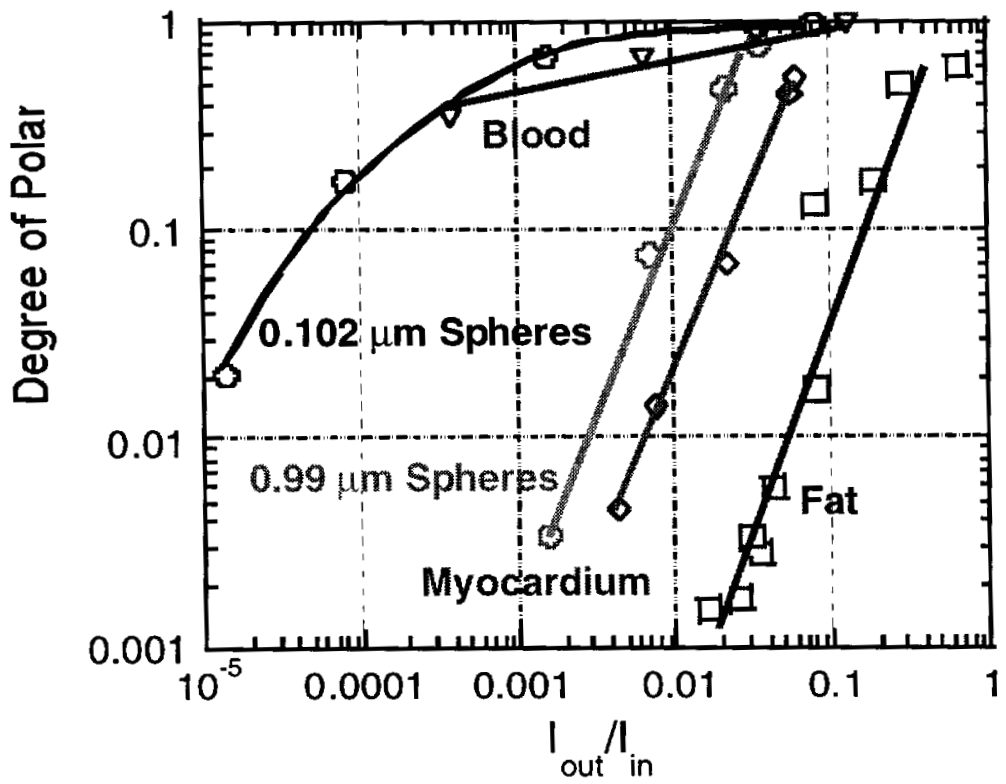

Figure 4. The linear degree of polarization is plotted against another dependent variable, intensity attenuation, for three tissues and two dilute phantoms. 


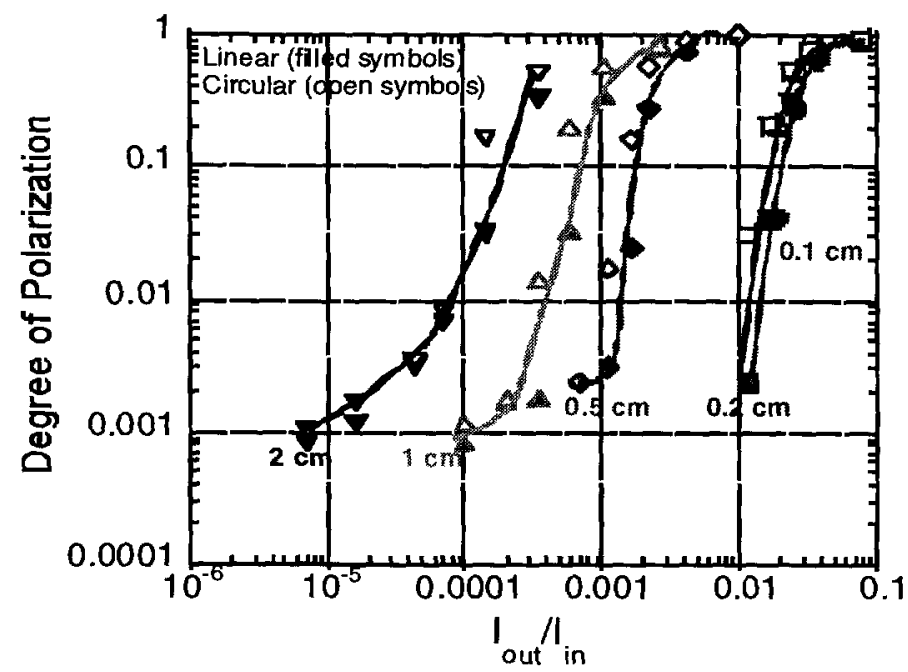

Figure 5. Phantoms can be made to span a large area of the degree of polarization versus intensity attenuation space. This example shows dilute phantoms of $0.99 \mu \mathrm{m}$ spheres with indicated pathlengths and varying densities within each pathlength curve.

Even though the dilute phantoms could be made to mimic the polarization/intensity characteristics, the differential scattering and depolarization of linear versus circular polarization states is also an important factor to consider in describing our degree of polarization results. Three different cases can be identified: $\mathrm{p}_{\mathrm{L}}>\mathrm{p}_{\mathrm{C}}$ (typical of scattering by dilute Rayleigh spheres), $\mathrm{p}_{\mathrm{L}}<$ $\mathrm{p}_{C}$ (typical of scattering by dilute Mie spheres), and $\mathrm{p}_{\mathrm{L}} \sim \mathrm{p}_{\mathrm{C}}$ (typical of scattering in the Rayleigh-Mie transition regime). Blood consists of dilute, large scatterers with size parameters that place them into the Mie regime. The results seen in blood demonstrate a preferential depolarization of linear over circular polarization states, also seen in the results for the dilute, Miesized tissue phantoms. Thus, the dilute Mie-sized scatterers found in blood can be well modeled by dilute, Mie-sized polystyrene spheres. Myocardial tissue consists of densely packed structures with a distribution of sizes that span the Rayleigh and Mie scattering regimes but are mostly Mie-sized. However, the results seen in the muscle tissue exhibit a preferential depolarization of circular over linear polarization states, which is typical of scattering by dilute Rayleigh spheres. The reasons why Rayleigh-like depolarization is seen for scattering by dense Mie-sized scatterers in this tissue are likely a combined effect of scatterer size and density. Thus, the effect of scatterer density for dilute and dense concentrations of spheres was studied next.

\subsection{TISSUE PHANTOMS: DENSITY EFFECT}

The effects of scatterer density was studied using aqueous suspensions of polystyrene microspheres with diameters of 0.48 and $0.99 \mu \mathrm{m}$, both of which are Mie-sized spheres. A wide range of sphere concentrations was studied so that depolarization by independently and dependently scattering spheres could be studied. For dilute concentrations of each suspension, as the scatterer concentration increases the degree of linear and circular polarization both decrease. Linearly polarized light is depolarized more quickly than circularly polarized light, a result corroborated both by the results shown in Figure 4 and by reports in the literature $(13,14)$. Above a critical concentration of scatterers, the degree of linear and circular polarization begin to increase with increasing scatterer concentration. The concentration at which there is an inflection in the degree of polarization follows trends seen in previous studies that report changes in unpolarized light attenuation through densely packed microsphere suspensions $(15,16)$. For these dense suspensions, circularly polarized light is depolarized similar to or more quickly than linearly polarized light, comparable to results seen in both the dilute suspensions of smaller $(0.102-\mu \mathrm{m}-$ diamter) spheres and the myocardial tissue. 


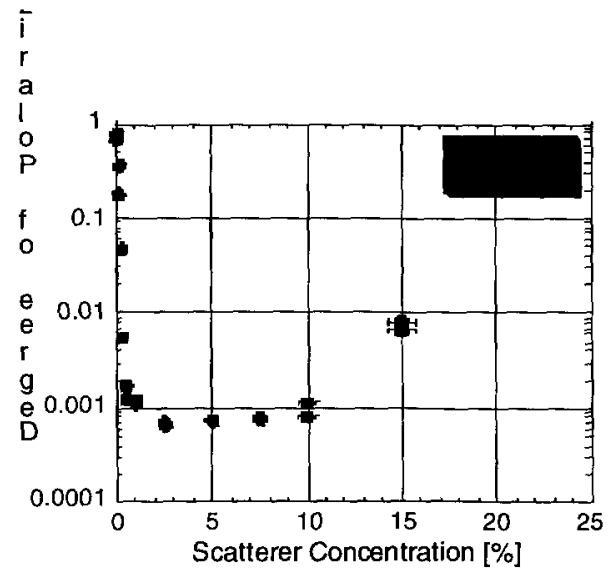

(a)

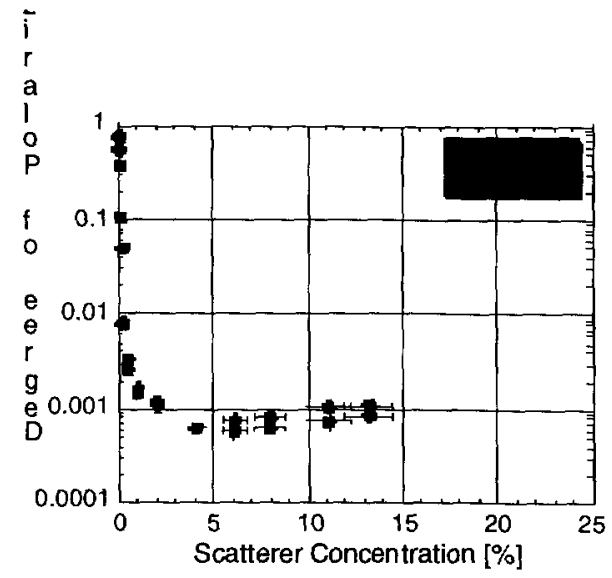

(b)

Figure 5. The degree of polarization was measured as a function of scatterer concentration in suspensions with sphere diameters of (a) $0.48 \mu \mathrm{m}$ and (b) $0.99 \mu \mathrm{m}$.

\section{CONCLUSIONS}

Polarized light propagation has typically been studied in tissue phantoms consisting of microspheres suspended in water. In common phantoms, such as dilute aqueous suspensions of $1-\mu \mathrm{m}$-diameter polystyrene spheres and Intralipid, it has been seen that circularly polarized light survives better than linearly polarized light $(9,14)$. However, we found in this study that similar behavior is not always seen in biologic tissue. In porcine blood, which consists of dilute, Mie scatterers, results similar to that seen in common phantoms were found; namely, circular polarization is maintained better than linear polarization. In porcine myocardial tissue, however, which consists of densely packed Rayleigh and Mie scatterers, the reverse was true. Linearly polarized light was maintained better than circularly polarized light. The reasons behind why different results were found in the two tissues may be attributed to the differences in the microstructure of the scatterers found in each case.

The effect of scatterer size on polarimetric measurements was first studied using aqueous suspensions of microspheres. Dilute concentrations were used so that independent scattering could be assumed. For small spheres that fell into the Rayleigh-Mie transition region, it was seen that linearly polarized light was maintained better than circularly polarized light. As the sphere size increased (i.e. Mie scattering), the reverse was true; circularly polarized light was maintained better than linearly polarized light. Thus, depending on the tissue to be modeled, a sphere size should be chosen for the phantom to mimic the differential depolarization of linear versus circular polarization states of the tissue under study. However, even though the resulting phantom may mimic the depolarization behavior of the tissue under limited conditions, it is unlikely that the structure of the phantom bears any semblance to the structure of the tissue. For example, even though blood and muscle exhibit differential depolarization behavior that is characteristic of Mie and Rayleigh scattering respectively, the physical structures in both tissues are predominantly Mie-sized. Thus, some factor other than sphere size must also be considered if the phantom material is to approach the microstructure of the tissue scatterers. One such factor is scatterer concentration, in a range outside of the independent scattering assumption made here.

Many tissues contain structures that are densely packed and thus cannot be well represented by dilute suspensions of spheres. Thus the next effect that was studied was scatterer concentration for both dilute and dense concentrations of spheres. We found that for dense suspensions of microspheres, polarized light propagation through a dense suspension of Mie spheres is similar to polarized light propagation through a dilute suspension of smaller Rayleigh spheres. Specifically, we observed that for dense concentrations of the 0.48 and $0.99-\mu \mathrm{m}$-diameter particles, each of which fall into the Mie scattering regime for dilute concentrations, circularly polarized light was depolarized similar to or more quickly than linearly polarized light. This corresponds to the behavior seen for scattering by dilute suspensions of particles in the Rayleigh-Mie and Rayleigh regions. Therefore myocardial tissue, which could be modeled by a suspension of dilute Rayleigh spheres, could perhaps be modeled better using a suspension of dense Mie spheres, which mimics the actual microstructure of the tissue more accurately. In understanding how polarized light propagates in biologic tissue, and in designing a phantom to mimic a particular tissue, it thus seems likely that both scatterer size and density (in a range outside of independent scattering) should be considered. 


\section{ACKNOWLEDGMENTS}

We thank the Affirmative Action and Diversity Program at Lawrence Livermore National Laboratory and the Department of Energy Center of Excellence for their support of this research. This study was performed under the auspices of the U.S. Department of Energy under Contract W-7405-ENG-48.

\section{REFERENCES}

${ }^{1}$ L.R. Bissonnette, "Imaging Through Fog and Rain," Opt. Eng. 31(5): 1045-1052 (1992).

${ }^{2}$ G.R. Fournier, D. Bonnier, J.L. Forand, and P.W. Pace, "Range-Gated Underwater Laser Imaging-System," Opt. Eng. 32(9): 2185-2190 (1993).

${ }^{3}$ K.M. Yoo, B.B. Das, and R.R. Alfano, "Imaging of a translucent object hidden in a highly scattering medium from the early portion of the diffuse component of a transmitted ultrafast laser pulse," Opt. Lett. 17(13), 958-960 (1992).

${ }^{4}$ B.J. Tromberg, L.O. Svaasand, T.-T. Tsay, and R.C. Haskell, "Properties of photon density waves in multiple-scattering media," Appl. Opt. 32, 607-616 (1993).

${ }^{5}$ B. Devaraj, M. Usa, K.P. Chan, and T. Akatsuha, "Recent Advances in Coherent Detection Imaging (CDI) in Biomedicine: Laser Tomography of Human Tissues In Vivo and In Vitro," IEEE J. Selected Topics in Quantum Elec. 2(4), 1008-1016 (1996).

${ }^{6}$ D.S. Dilworth, E.N. Leith, and J.L. Lopez, "Imaging absorbing structures within thick diffusing media," Appl. Opt. 29, 691-698 (1990).

${ }^{7}$ J.M. Schmitt, A.H. Gandbakhche, and R.F. Bonner, "Use of polarized light to discriminate short-path photons in a multiply scattering medium," Appl.Opt. 31(30), 6535-6546 (1992).

${ }^{8}$ O. Emile, F. Bretenaker, and A. Le Floch, "Rotating polarization imaging in turbid media," Opt. Lett. 21(20), 1706-1708 (1996)

${ }^{9}$ V. Sankaran, M.J. Everett, D.J. Maitland, and J.T. Walsh, Jr. "Comparison of polarized light propagation in biologic tissue and phantoms," Op. Lett. 24, 1044-1046 (1999).

${ }^{10}$ M. Kerker, The Scattering of Light and other electromagnetic radiation (Academic Press, Inc., San Diego, 1969).

${ }^{11}$ R.G. Kessel, Basic Medical Histology: The Biology of Cells, Tissues, and Organs (Oxford University Press, New York, 1998).

12 J.A.G. Rhodin, Histology A Text and Atlas (Oxford University Press, New York, 1974).

${ }^{13}$ F.C. MacKintosh, J.X. Zhu, D.J. Pine, and D.A. Weitz, "Polarization memory of multiply scattered light," Phys. Rev. B 40(13), 9342-9345 (1989).

${ }^{14}$ D. Bicout, C. Brosseau, A.S. Martinez, and J.M. Schmitt, "Depolarization of multiply scattered waves by spherical diffusers: Influence of the size parameter," Phys. Rev. E 49(2), 1767-1770 (1994).

${ }^{15}$ V.P. Dick, "Applicability of limits of Beer's law for dispersion media with a high concentration of particles," Appl. Opt. 37, 4998-5004 (1998). 
${ }^{16}$ A. Ishimaru and Y. Kuga, "Attenuation Constant of a Coherent Field in a Dense Distribution of Particles," J. Opt. Soc. Am. 72, 1317-1320 (1982). 
3. MEDLINE/HealthSTAR 1990-1994

Patrick, JT; Nolting, MN; Goss, SA; Dines, KA; Clendenon, JL; Rea, MA

Heimburger, RF. Ultrasound and the blood-brain barrier.

Advances in Experimental Medicine and Biology, 1990, 267:369-81, (UI)

91206153

Language: English; Pub type: JOURNAL ARTICLE

Abstract: High intensity focused ultrasound was employed to modify the

permeability of the normal feline and canine blood-brain barrier (BBB) to a

circulating vital dye--Evans blue (EB). The threshold doses (W sec/cm2) for

focally increasing the permeability of the $\mathrm{BBB}$ in white matter (WM) and gray

matter (GM) were as follows: internal capsule (WM) -340 to 680 ; thalamus

(GM)--approximately 1326; and caudate nucleus (GM)--2284 to 2952. In the

presence of supralesioning doses of ultrasound, the cross sectional area

occupied by the EB was consistently greater than that of the attendant

nonhemorrhagic lesion--thus suggesting that $B B B$ changes may be inducible at

sublesioning doses. These findings, in conjunction with those of others.

suggest that high intensity focused ultrasound may have a role in the

treatment of brain tumors based on cell destruction by two mechanisms: (a)

direct, by the ultrasound and (b) indirect, by an antineoplastic agent which

is delivered via an ultrasonically modified BBB. 